\title{
Dyschromatosis universalis hereditaria: A rare case report from Northeast India
}

\section{Romita Bachaspatimayum, Priyanka Das, Nandita Bhattacharjee}

Department of Dermatology, Venereology and Leprology, Regional Institute of Medical Sciences, Imphal, Manipur, India

Corresponding author: Dr. Priyanka Das, E-mail: iampriyanka1243@gmail.com

\begin{abstract}
Dyschromatosis Universalis Hereditaria is a rare genodermatoses characterised by hyperpigmented and hypopigmented macules which are variable in size and shape. We report a case of Dyschromatosis Universalis Hereditaria from North east India who presented with multiple asymptomatic hyperpigmented and hypopigmented lesions over trunk and extremities since birth with positive family history which was later confirmed by histopathology.
\end{abstract}

Key words: Dyschromatosis universalis hereditaria; Genodermatoses; Pigmentary disorder

\section{INTRODUCTION}

DUH is a rare genodermatoses characterised by hyperpigmented and hypopigmented macules which are variable in size and shape [1]. It is a spectrum of diseases which includes dyschromatosis universalis hereditaria (generalized form), dyschromatosis symmetrica hereditaria (localized form) and unilateral dermatomal pigmentary dermatosis (segmental form) [2]. Dyschromatosis universalis hereditaria was first described by Ichikawa and Hiraga in 1933 [3].

\section{CASE REPORT}

An 18 years old muslim unmarried male, manual labourer by profession, presented with chief complaints of multiple asymptomatic hyperpigmented and hypopigmented lesions over trunk and extremities since birth. The lesions were present over chest and lower legs by birth while it spread to thighs, abdomen, back of trunk, arms and forearms by 3 years of age. There were no history of photosensitivity, handling chemicals and intake of any significant drugs. No history suggestive of seizure disorder was reported. Similar lesions were present in his paternal aunt since the age of 2 years. He was borne of a non - consanguineous marriage.
Dermatological examination revealed numerous hyperpigmented macules interspersed with hypopigmented macules of irregular shape and 0.5 to $2 \mathrm{~cm}$ in size distributed over neck, trunk and extremities (Fig. 1). Face, palms and soles were spared. Mucosal surfaces, hair and nail were normal. There was no associated telangiectasia and atrophy.

Systemic examination did not reveal any abnormality. There was no associated short stature, cataract or deafness. Routine laboratory investigations including VDRL and HIV test were negative.

Skin biopsy from hyperpigmented lesion showed orthokeratotic epidermis and increase in melanin content. Biopsy from hypopigmented lesions showed decrease in melanin content and yeast in stratum corneum (Fig. 2).

\section{DISCUSSION}

Dyschromatosis universalis hereditaria is a rare pigmentary disorder which encompasses both hypo and hyperpigmented macules coalescing to form a reticular pattern [4]. The majority of cases of dyschromatosis universalis hereditaria show autosomal dominant pattern but some cases with autosomal recessivepattern have also been reported and the locus has been mapped

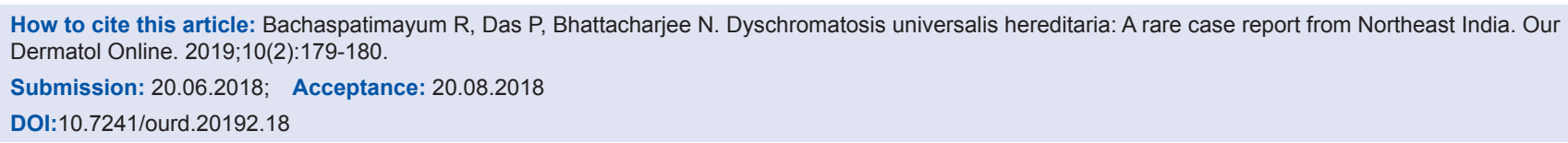




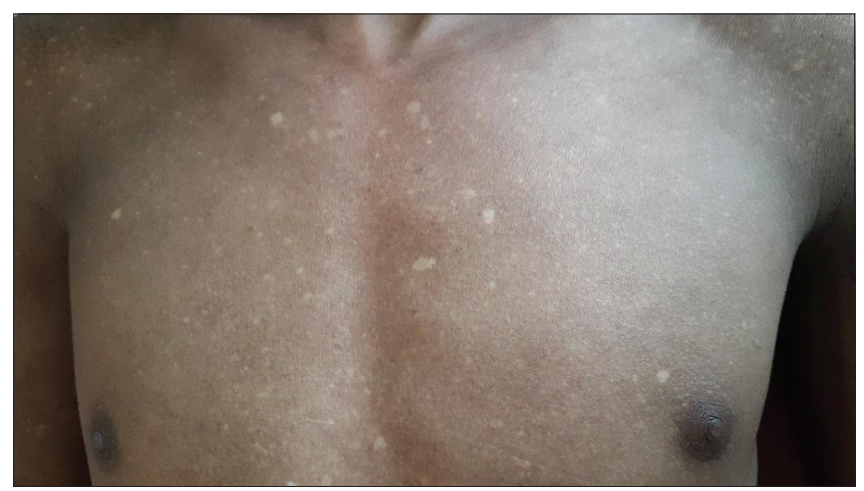

Figure 1: Numerous hyperpigmented macules interspersed with hypopigmented macules of irregular shape and 0.5 to $2 \mathrm{~cm}$ in size distributed over neck \& trunk.

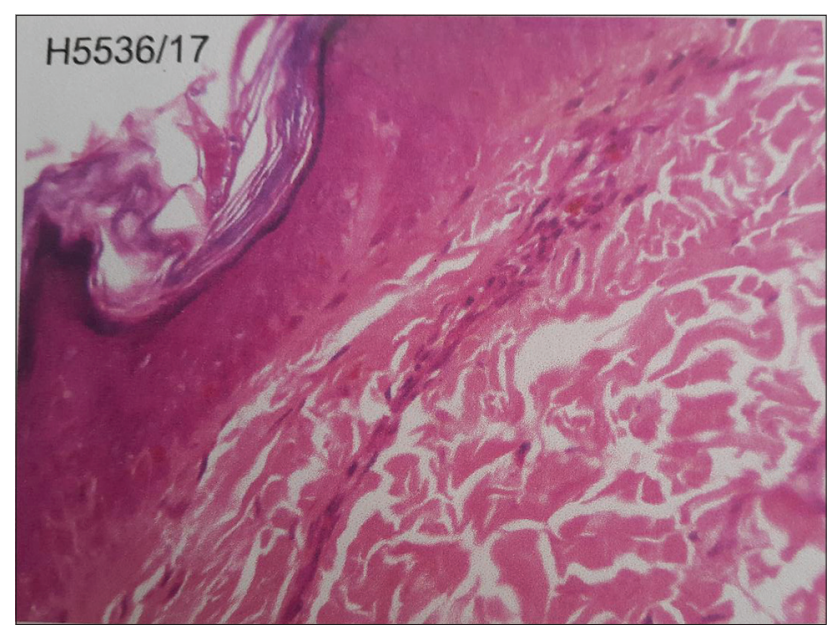

Figure 2: Skin biopsy from hyperpigmented lesions showed orthokeratotic epidermis and increase in melanin content with many scattered melanophages in upper dermis.

to chromosome 6q24.2eq25.2 and 12q21eq23 [5]. In our case, patient's paternal aunt was also having similar complaints in milder form. In a recent ultrastructural study, it has been suggested that DUH is a disorder of melanosome production rate or melanosome activity, rather than a disorder of melanocyte number [6]. Most cases present early in life [7]. But in our case the lesions were present since birth. The trunk and the extremities are commonly involved. Face is rarely involved and palms, soles and mucous membranes are spared [8]. Various systemic abnormalities have been described with DUH. These include Dowling-Degos disease, X-linked ocular albinism, tuberous sclerosis, abnormalities in erythrocyte, platelet and tryptophan metabolism, epilepsy, insulin-dependent diabetes mellitus, ocular abnormalities, photosensitivity, learning difficulties, mental retardation $[9,10]$.

Lesions of dyschromatosis universalis hereditaria (DUH) have to be differentiated from xeroderma pigmentosum since in both cases photoexposed areas are involved. However, in our patient lesions were present in unexposed areas as well and there were no atrophy or telangiectasia. The other differential diagnoses of DUH are dermatopathia pigmentoreticularis, NaegeliFranceschetti-Jadassohn syndrome and dyskeratosis congenita (DKC).There is no effective treatment modality till date [8].

\section{CONCLUSION}

Previously it was thought that DUH occurs only in Japanese population. But recently it has been frequently reported in other races as well. Few cases have been reported from India. But there is no documented case report from north east India till now to the best of our knowledge. We are reporting this case because of its rarity.

\section{CONSENT}

The examination of the patient was conducted according to the Declaration of Helsinki principles.

\section{REFERENCES}

1. Naik CL, Singh G, Rajashekar TS, Okade R. Dyschromatosis universalis hereditaria. Indian J Dermatol. 2009;54:74-5.

2. Yadalla HK, Pinninti S, Babu AR. Dyschromatosis universalis hereditaria: Infrequent genodermatoses in India. Indian J Hum Genet. 2013;19:487-90.

3. Ichikawa T, Higara Y. About a pigmentary anomaly unprecedented. Jpn J Dermatol. 1933;34:360-4.

4. Stuhrmann M, Hennies H, Bukhari I, Brakensiek K, Nürnberg G, Becker C, et al. Dyschromatosis universalis hereditaria: evidence for autosomal recessive inheritance and identification of a new locus on chromosome 12q21-q23. Clin Genet. 2008;73:566-72.

5. Doss RW. Dyschromatosis in a Child-A Case Report and differential Diagnosis. Clin Pediatr Dermatol. 2016;2:1.

6. Nuber UA, Tinschert S, Mundlos S, Hauber I. Dyschromatosis universalis hereditaria: Familial case and ultrastructural skin investigation. Am J Med Genet A. 2004;125:261-6.

7. Udayashankar C, Nath AK. Dyschromatosis universalis hereditaria: A case report. Dermatol Online J. 2011;17:2.

8. Namitha P, Sacchidanand S. Dyschromias: A series of five interesting cases from India. Indian J Dermatol. 2015;60:636-8.

9. Binitha MP, Thomas D, Asha LK. Tuberous sclerosis complex associated with dyschromatosis universalis hereditaria. Indian J Dermatol Venereol Leprol. 2006;72:300-2.

10. Al Hawsawi K, Al Aboud K, Ramesh V, Al Aboud D. Dyschromatosis universalis hereditaria: Report of a case and review of the literature. Pediatr Dermatol. 2002;19:523-6.

Copyright by Romita Bachaspatimayum, et al. This is an open-access article distributed under the terms of the Creative Commons Attribution License, which permits unrestricted use, distribution, and reproduction in any medium, provided the original author and source are credited.

Source of Support: Nil, Conflict of Interest: None declared. 